\title{
Lumbar hernia repair: Myth or reality?
}

\author{
Aziz Sümer, ${ }^{1}$ Ediz Altınlı, ${ }^{2}$ Ersan Eroğlu, ${ }^{2}$ Mehmet Ali Uzun, ${ }^{3}$ Serkan Senger ${ }^{3}$ \\ 'Department of General Surgery, Yuzuncu Yil University Faculty of Medicine, Van, Turkey \\ ${ }^{2}$ Department of General Surgery, Istanbul Bilim University Faculty of Medicine, Istanbul, Turkey \\ ${ }^{3}$ Department of General Surgery, Haydarpaşa Numune Training and Research Hospital, Istanbul, Turkey
}

\begin{abstract}
Introduction: The purpose of this study was to present and share experience with lumbar hernia repair.

Materials and Methods: Between 2004 and 2013, 5 lumbar hernia repairs were performed. Mean follow-up period was 41 months, and no recurrence was encountered.

Results: Following the repair, results were good with respect to body balance, but cosmetic results, even with laparoscopic approach, were questionable.

Conclusion: Lumbar hernia should be repaired, but results are not 100\% reliable. Patients will have good results for body balance following the repair, but cosmetic results, even in laparoscopic approach, are less satisfactory.

Keywords: Lumbar hernia repair.
\end{abstract}

\section{Introduction}

Lumbar hernia defines the defects localized on the posterolateral abdominal wall. Lumbar hernias are uncommon defects. Three hundred cases have been reported to date in the current literature..$^{[1]}$

Although many surgical techniques have been proposed for the management of these types of hernias, none of them has been recommended as the gold standard method. Because of its rarity, there has been difficulty in defining the margins of the defect, the presence of a bone limits operative maneuvers, concomitant paralysis of the muscles is a complicating factor, and there is a lack of sufficient experience among surgeons. ${ }^{[2]}$

The purpose of this study is to present and share our experience in lumbar hernia repair.

\section{Materials and Methods}

Between 2004 and 2013, a total of five patients underwent lumbar hernia repair operation in our institution. Demographic data, hernia type, etiology of the hernia, operation type, length of hospital stay, and morbidity and mortality rates were evaluated retrospectively. The diagnosis was determined based on the clinical suspicion of lumbar hernia. For the differential diagnosis, conventional abdominal X-ray, ultrasound (US) and computed tomography (CT) were used (Figure 1).

\section{Results}

Three patients were male $(60 \%)$ and two were female (40\%). The mean age was 49 years (range, 25-71 years). Etiology of the hernia was determined as Grynfeltt hernia in one patient, incisional hernia in three patients 


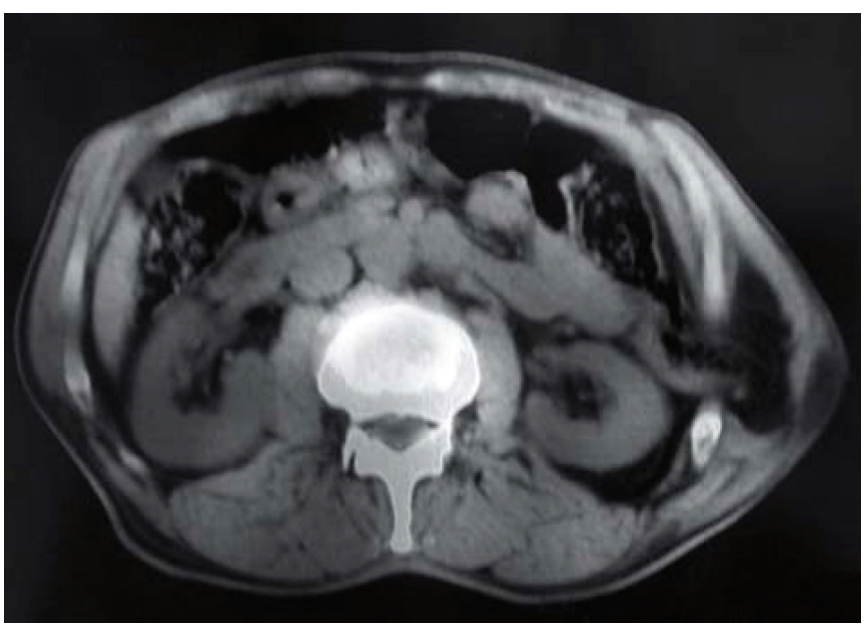

Figure 1. Computed tomography of abdomen shows left posterolateral abdominal wall defect, including small intestine.

and traumatic lumbar hernia in one patient. Grynfeltt hernia was operated with an initial diagnosis of lipoma, and the hernia was found perioperatively to be Grynfeltt type.

For incisional hernia cases, laparoscopic intraperitoneal mesh repair was performed. In Grynfeltt hernia, open primary closure was done. For the traumatic lumbar hernia case, open mesh hernioplasty was applied.

In one case, following laparoscopic intraperitoneal mesh repair, seroma formation was encountered in the early postoperative period. We performed re-laparoscopy and punctured the mesh with Veress needle in order to resolve seroma.

The average length of hospital stay was 3 days (range, 1-7 days). There was no mortality. The data including patient demographics, treatment methods, morbidity, mortality, and length of hospital stay are shown in Table 1.

The mean follow-up period was 41 months, and no recurrences were encountered.

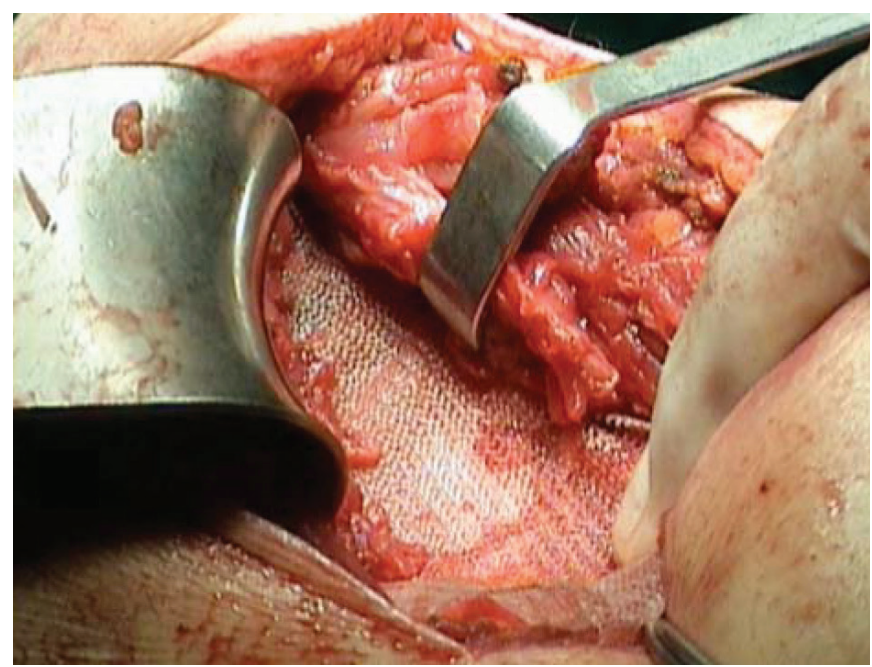

Figure 2. Full-thickness mesh fixation with polypropylene sutures.

\section{Surgical Techniques}

Open surgical approach for traumatic lumbar hernia: With skin incision, an $8 \mathrm{~cm}$ defect was detected between the 10th rib and external oblique muscle, and herniation of intestinal loops from this defect was observed. The peritoneum was closed with Vicryl sutures, and a 10x10 $\mathrm{cm}$ polypropylene mesh was placed over the defect with significant overlap on all sides. The mesh was secured in an extra-peritoneal location with full-thickness polypropylene sutures (Figure 2). The skin was closed following aspirative drain insertion under the skin. The drain was removed on the third postoperative day.

Open surgical approach for Grynfeltt hernia: Surgery was performed by direct approach. After the skin incision, the layers were easily dissected, and the bulged transversus abdominis aponeurosis was found. After opening the transversus abdominis aponeurosis, the fat responsible for this protrusion was easily reintegrated through the small $2.5 \mathrm{~cm}$ defect. The wall was reinforced by a nonabsorbable running suture between the internal oblique

\section{Table 1. Patient data}

\begin{tabular}{lccccccc} 
N & Gender & Age (year) & Hernia type & Treatment modality & Complication & Mortality & LHS \\
\hline 1 & Male & 71 & Traumatic lumbar & Open (mesh) repair & None & None & 4 \\
2 & Female & 55 & Incisional & Laparoscopic repair & Seroma & None & 7 \\
3 & Male & 25 & Grynfeltt & Open repair & None & None & 1 \\
4 & Male & 51 & Incisional & Laparoscopic repair & None & None & 1 \\
5 & Female & 43 & Incisional & Laparoscopic repair & None & None & 2 \\
\hline \multicolumn{7}{l}{ N: Number of patient; LHS: Length of hospital stay (day). }
\end{tabular}


muscle, transversus abdominis aponeurosis, and latissimus dorsi muscle and lumbocostal ligament.

Laparoscopic approach for incisional hernia: Under intratracheal general anesthesia, the patient was placed in a full lateral decubitus position. In order to optimize exposure and better open the space between the rib cage and the iliac crest, a lumbar roll was placed under the lumbar region. One $10 \mathrm{~mm}$ and two $5 \mathrm{~mm}$ trocars were used during the operation (Figure 3). The first trocar was placed using open Hasson technique. Then, two $5 \mathrm{~mm}$ trocars were inserted under direct vision. All trocars were placed in the midline position at least $5 \mathrm{~cm}$ apart. Pneumoperitoneum was established with carbon dioxide at an average of $12 \mathrm{mmHg}$. A $30^{\circ}$ laparoscope was used during the whole procedure. After exploration of the abdominal cavity, omental adhesions from the previous surgeries were dissected free, exposing the hernia defect (Figure 4). The contents of the hernia were carefully extracted from the sac, and adhesions of these contents to the sac were divided as needed. The hernia size was measured, and the mesh was adjusted to its size with at least a $4 \mathrm{~cm}$ margin in all directions. Dual mesh was used for repair. Mesh sizes were $20 \times 30 \mathrm{~cm}$ and $15 \times 20 \mathrm{~cm}$ for the two cases.

Marks were made on the mesh and on the external abdominal surface to assist with intra-abdominal orientation. After sutures were placed on the four corners of the mesh, it was wrapped around a laparoscopic grasper and inserted through the $10 \mathrm{~mm}$ trocar site. Once the mesh was unrolled and placed in the correct position, the preplaced sutures were pulled through the abdominal wall with the help of a suture passer (Figure 5). The mesh was fixed with double crown technique using helical fasteners.

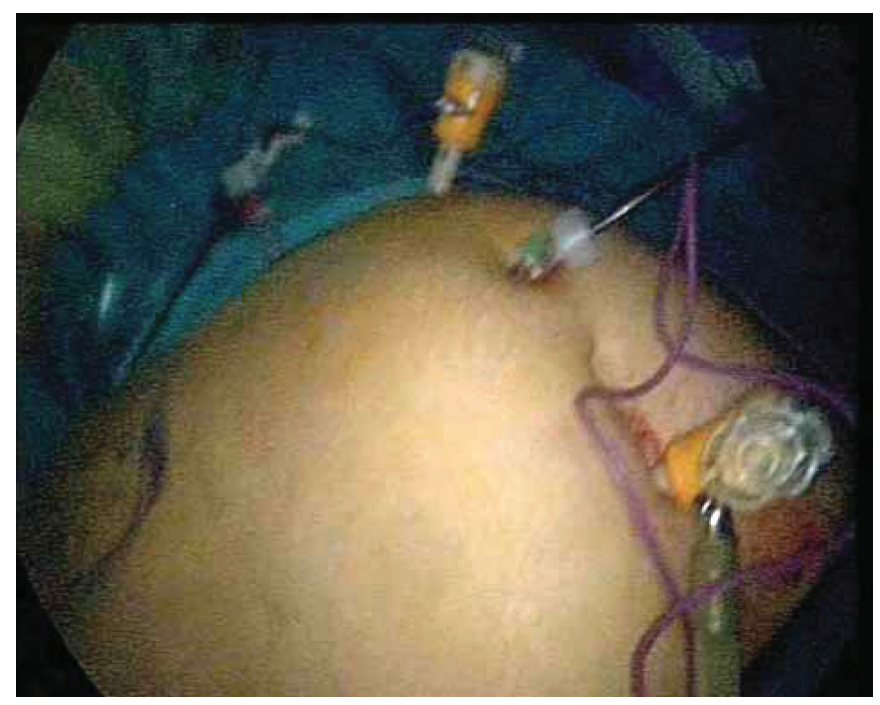

Figure 3. Placement of three trocars in the midline.

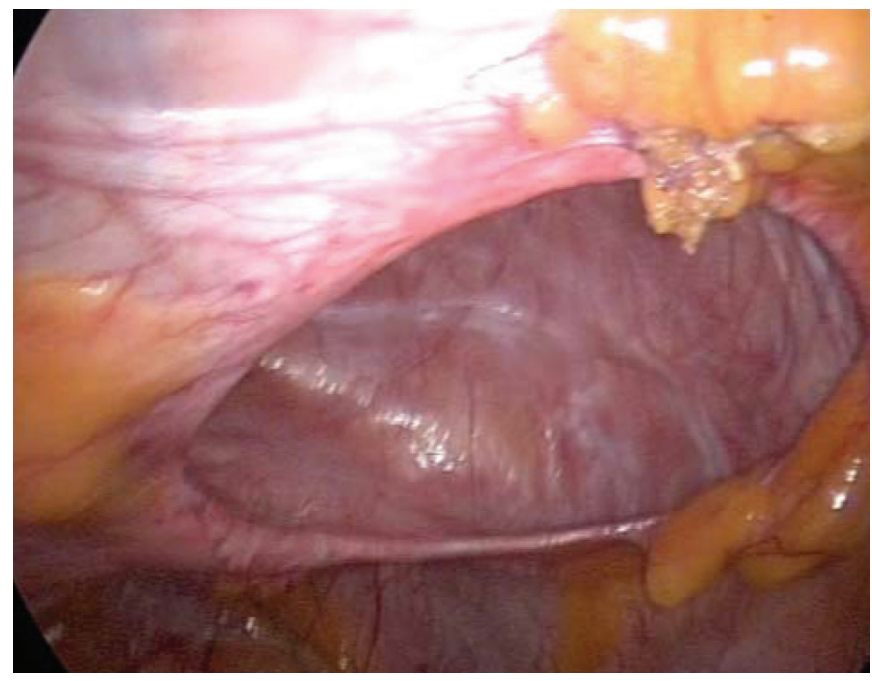

Figure 4. Hernia defect view.

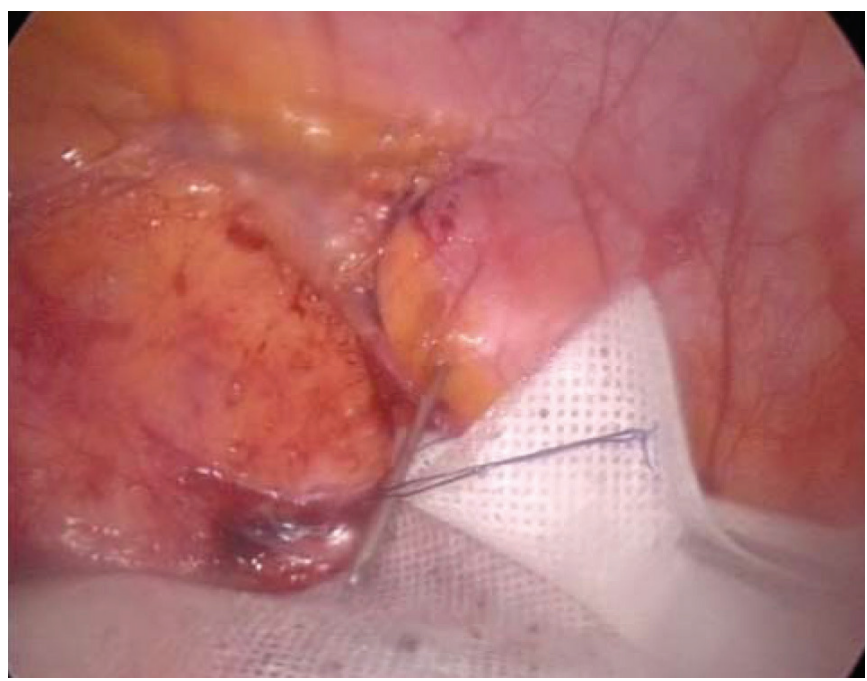

Figure 5. Positioning of the mesh with the help of suture passer.

\section{Discussion}

After the laparoscopic approach was shown to be beneficial for ventral hernia repair, a similar technique was developed for other defects of the abdominal wall. ${ }^{[2]}$ Lumbar hernia is one of the lesser-known hernias occurring through the posterior abdominal wall. Its incidence is not more than $2 \%$ of all abdominal hernias. ${ }^{[3]}$

In 2009, the European Hernia Society developed a classification system according to localization and size of the hernia. In this classification system, lumbar hernia was defined as the $\mathrm{L} 4$ area of the abdominal wall. The borders of the lumbar region are defined as the $12^{\text {th }}$ rib superiorly, iliac crest inferiorly, anterior axillary line anteriorly, and erector spinae muscle posteriorly. ${ }^{[4]}$

Lumbar hernias are classified as congenital and acquired 
hernias according to their etiology. Acquired hernias account for $80 \%$ of lumbar hernias. Acquired lumbar hernias were divided into two groups as spontaneous and secondary lumbar hernia. Etiologic factors causing spontaneous hernias are increased intraabdominal pressure, obesity, elder age, and diseases causing muscular atrophy, such as polio. Etiologic factors for secondary lumbar hernia are trauma, surgery and inflammation. ${ }^{[5]}$ In the current study, one patient had traumatic lumbar hernia, one had congenital Grynfeltt hernia and three had incisional hernia due to previous nephrectomies. Lumbar hernia can be seen after laparoscopic and open nephrectomies, repair of abdominal aorta aneurysm and giant abdominal wall mass excision. ${ }^{[1]}$ An interesting acquired lumbar hernia is believed to be from a case of herpes zoster exacerbation that resolved after resolution of the herpetic lesions. ${ }^{[6]}$

The management of lumbar hernia is controversial. The main questions in its management are "when?" and "how?". When is surgery indicated? The natural history of lumbar hernias is progressive. Therefore, most authors believe that the hernia should always be repaired, except in high-risk patients. Because surgical correction is al- ways more difficult in advanced cases, surgery should be indicated as early as possible..$^{[2,7]}$

How should lumbar hernia be repaired? The debate of open versus laparoscopic repair of lumbar hernias is ongoing. ${ }^{[2]}$ Both transabdominal pre-peritoneal and totally extra pre- peritoneal techniques are used in laparoscopic repair of lumbar hernia. The first laparoscopic lumbar hernia repair was defined by Burick et al. ${ }^{[8]}$

The disadvantages of the open approach include difficulty in operation due to fascial attenuation and bony hernia boundaries including the iliac crest and/or $12^{\text {th }}$ rib and the lack of adequate tissue for coverage of extensive dissection. This approach also requires a large incision and may result in significant postoperative morbidity. The bone boundaries established for the hernia defect may also make adequate fixation of the synthetic material difficult. ${ }^{[9]}$

In the current study, the open approach was used for the congenital and traumatic lumbar hernia cases. Traumatic lumbar hernia was first reported by Selby in 1906. ${ }^{[10]} \mathrm{Al}-$ though traumatic lumbar hernia is a rare entity, the most common causative factor is motor vehicle accident. How-

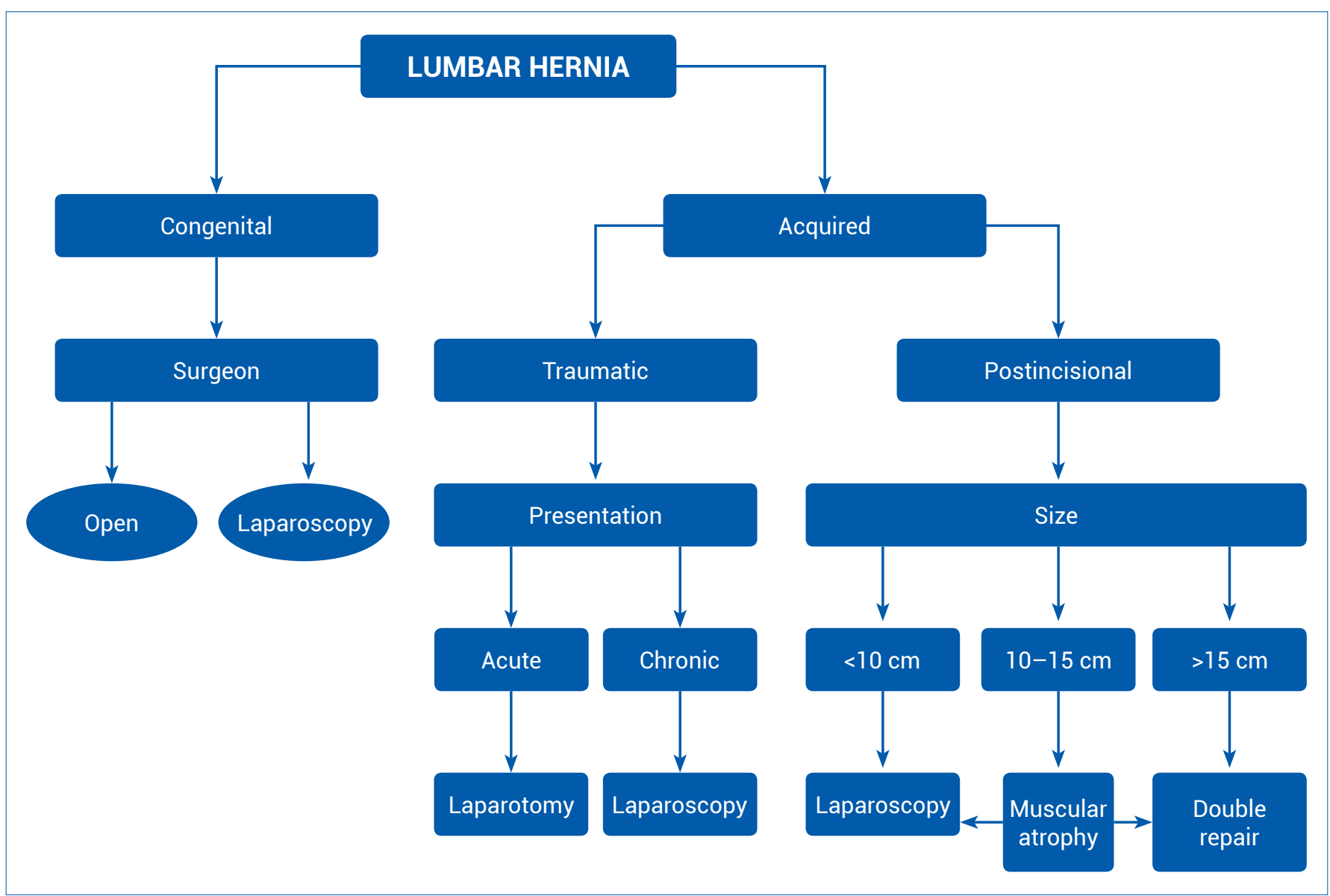

Figure 6. The current algorithm for lumbar hernia. ${ }^{[14]}$ 
ever, fall from height (4 meters) was the causative factor in one of our cases.

In the lumbar area, the laparoscopic approach seems to offer certain advantages, enabling identification of the entirety of the lumbar area, accurate evaluation of the hernia type, complete reconstruction of the area, and the placement of a mesh that amply overlaps the margins of the defect, including the bone. Arca et al., ${ }^{[11]}$ Heniford et al., ${ }^{[12]}$ and Bickel et al. ${ }^{[13]}$ recommend the laparoscopic technique as the method of choice for lumbar hernia repair for reasons of its simplicity and safety, and the quick recovery of the patient. In our study, we performed three laparoscopic intraperitoneal mesh repairs. Because of flaccid paralysis after lumbotomy incision, the cosmetic result after laparoscopic repair of incisional lumbar hernia is also questionable. However, the repair of this type hernia is necessary for re-establishment of body balance.

The biggest case load of lumbar hernia repair was reported by Morena-Egea et al. ${ }^{[14]}$ In their study, they showed the risk factors for recurrence to be associated with localization and defect size. Diffuse lumbar hernias had a recurrence rate of $42.9 \%$. They pointed out some important deductions related with lumbar hernias, as follows: 1 ) The laparoscopic approach is associated with less operating time, shorter hospital stays, an earlier return to normal activity, and lower analgesic consumption. 2) Open surgery may be considered the best option in diffuse hernias with defects larger than $15 \mathrm{~cm}$. 3) The lightweight mesh does not increase the recurrence rate in lumbar hernia repair. The current algorithm is shown in Figure 6.

In conclusion, lumbar hernias should be repaired, but the results are not $100 \%$ reliable. Patients will have good results for body balance following the repair, but the cosmetic results of the repair, even in the laparoscopic approach, are questionable.

\section{References}

1. Gagner M, Milone L, Gumbs A, Turner P. Laparoscopic repair of left lumbar hernia after laparoscopic left nephrectomy. JSLS 2010;14:405-9.

2. Moreno-Egea A, Torralba-Martinez JA, Morales G, Fernández T, Girela E, Aguayo-Albasini JL. Open vs laparoscopic repair of secondary lumbar hernias: a prospective nonrandomized study. Surg Endosc 2005;19:184-7.

3. Armstrong O, Hamel A, Grignon B, NDoye JM, Hamel O, Robert $\mathrm{R}$, et al. Lumbar hernia: anatomical basis and clinical aspects. Surg Radiol Anat 2008;30:533-7.

4. Muysoms FE, Miserez M, Berrevoet F, Campanelli G, Champault GG, Chelala $E$, et al. Classification of primary and incisional abdominal wall hernias. Hernia 2009;13:407-14.

5. Hide IG, Pike EE, Uberoi R. Lumbar hernia: a rare cause of large bowel obstruction. Postgrad Med J 1999;75:231-2.

6. Hindmarsh A, Mehta S, Mariathas DA. An unusual presentation of a lumbar hernia. Emerg Med J 2002;19:460.

7. Uzun MA, Köksal N, Onur E, Günerhan Y, Sahin UY, Celik A. Traumatic lumbar hernia. Ulus Travma Acil Cerrahi Derg 2008; 14:253-5.

8. Burick AJ, Parascandola SA. Laparoscopic repair of a traumatic lumbar hernia: a case report. J Laparoendosc Surg 1996;6:259-62.

9. Yavuz N, Ersoy YE, Demirkesen O, Tortum OB, Erguney S. Laparoscopic incisional lumbar hernia repair. Hernia 2009;13:281-6.

10. Selby CD. Direct abdominal hernia of traumatic origin. JAMA 1906;47:1485-6.

11. Arca MJ, Heniford BT, Pokorny R, Wilson MA, Mayes J, Gagner M. Laparoscopic repair of lumbar hernias. J Am Coll Surg 1998;187:147-52.

12. Heniford BT, lannitti DA, Gagner M. Laparoscopic inferior and superior lumbar hernia repair. Arch Surg 1997;132:1141-4.

13. Bickel A, Haj M, Eitan A. Laparoscopic management of lumbar hernia. Surg Endosc 1997;11:1129-30.

14. Moreno-Egea A, Alcaraz AC, Cuervo MC. Surgical options in lumbar hernia: laparoscopic versus open repair. A long-term prospective study. Surg Innov 2013;20:331-44. 